\title{
ON POSITIVE SOLUTIONS FOR A CLASS OF INDEFINITE WEIGHT SEMILINEAR ELLIPTIC BOUNDARY VALUE PROBLEMS WITH CRITICAL SOBOLEV EXPONENT
}

\author{
Bongsoo Ko and SeungPil Kang
}

\begin{abstract}
By variational methods, we prove the existence of positive solutions of a class of indefinite weight semilinear elliptic boundary value problems on critical Sobolev exponent.
\end{abstract}

\section{Introduction}

We have known several famous results for the existence or the non-existence of positive solutions about semilinear elliptic boundary value problems in critical Sobolev exponent case ([5], [2]). As some different studies from that, we discuss the existence of positive solutions of the following indefinite weight semilinear elliptic boundary value problems:

$$
\left(I_{\lambda \alpha}\right)\left\{\begin{array}{l}
-\Delta u=\lambda g(x) u\left(1+|u|^{p}\right) \text { in } \Omega \\
(1-\alpha) \frac{\partial u}{\partial n}+\alpha u=0 \text { on } \partial \Omega
\end{array}\right.
$$

where $\lambda$ and $\alpha$ are real parameters, $\Omega$ is an open bounded domain in $\mathbb{R}^{N}$, $N \geq 3$, with the smooth boundary $\partial \Omega$. We shall consider the critical Sobolev exponent case $p=\frac{4}{N-2}$ and the function $g: \bar{\Omega} \longrightarrow \mathbb{R}^{1}$ is smooth and changes sign.

We proved the existence of positive solutions of the case $0<p<\frac{4}{N-2}([4])$. Here $\alpha \in(0,1)$ or $\int_{\Omega} g(x) d x \neq 0$ and $\alpha \in\left(\alpha_{0}, 0\right]$ for some constant $\alpha_{0}<0$. We used the constrained minimization method of the functional

$$
E_{\lambda}(u)=\int_{\Omega}|\nabla u|^{2}-\lambda \int_{\Omega} g u^{2}+\frac{\alpha}{(1-\alpha)} \int_{\partial \Omega} u^{2} d S_{x}
$$

on the constrained set

$$
\left\{u \in W^{1,2}(\Omega): \lambda \int_{\Omega} g \mid u^{p+2}=1\right\}
$$

Received February 5, 2004.

2000 Mathematics Subject Classification. 35J60, 35J20.

Key words and phrases. indefinite weight semilinear elliptic problems, critical Sobolev exponent, positive solutions, variational methods. 
to prove the existence if $\alpha \neq 1$. The other case can be proved by the similar method on the Sobolev space $W_{0}^{1,2}(\Omega)$. In this paper, we assume that if $\alpha=1$, the considering space is $W_{0}^{1,2}(\Omega)$.

If $p=\frac{4}{N-2}$, the above constrained set may not be weakly closed, and so we should find a different method to get positive solutions.

In Section 2, we show that a minimizing sequence of the functional which is induced by the weighted problem $\left(I_{\lambda \alpha}\right)$ : For $\alpha \in[0,1)$,

$$
J_{\lambda \alpha}(u)=\frac{1}{2} \int_{\Omega}|\nabla u|^{2}-\frac{\lambda}{2} \int_{\Omega} g u^{2}-\frac{\lambda}{p+2} \int_{\Omega} g|u|^{p+2}+\frac{\alpha}{2(1-\alpha)} \int_{\partial \Omega} u^{2} d S_{x}
$$

on the Nehari manifold:

$$
M_{\lambda \alpha}=\left\{u \in W^{1,2}(\Omega): u \neq 0,\left\langle J_{\lambda \alpha}^{\prime}(u), u\right\rangle=0\right\},
$$

where

$$
\left\langle J_{\lambda \alpha}^{\prime}(u), u\right\rangle=\int_{\Omega}|\nabla u|^{2}-\lambda \int_{\Omega} g u^{2}\left(1+|u|^{p}\right)+\frac{\alpha}{1-\alpha} \int_{\partial \Omega} u^{2} d S_{x},
$$

converges to a positive function in $W^{1,2}(\Omega)$ which is a classical positive solution of the problem $\left(I_{\lambda \alpha}\right)$ if $\lambda_{\alpha}^{-}<\lambda<\lambda_{\alpha}^{+}$, and $\lambda$ is near to either $\lambda_{\alpha}^{-}$or $\lambda_{\alpha}^{+}$, where $\lambda_{\alpha}^{-}$and $\lambda_{\alpha}^{+}$are the principal eigenvalues of the following problem ([1]):

$$
\left(L_{\alpha}\right)\left\{\begin{array}{l}
-\Delta u=\lambda g(x) u \text { in } \Omega, \\
(1-\alpha) \frac{\partial u}{\partial n}+\alpha u=0 \text { on } \partial \Omega .
\end{array}\right.
$$

Furthermore, we estimate the length of the intervals about $\lambda$ in which the existence is guaranteed. We also have the similar result for $\alpha=1$ using the following functional

$$
J_{\lambda 1}(u)=\frac{1}{2} \int_{\Omega}|\nabla u|^{2}-\frac{\lambda}{2} \int_{\Omega} g u^{2}-\frac{\lambda}{p+2} \int_{\Omega} g|u|^{p+2} .
$$

In the end of Section 2 , we can show that $\left(I_{\lambda \alpha}\right)$ has a positive solution for all $\lambda \in\left(\lambda_{\alpha}^{-}, \lambda_{\alpha}^{+}\right)$, except $\lambda \neq 0$ if $g(x)<0$ for all $x \in \partial \Omega$. However, we note that if $\Omega$ is a ball, $g=1, N=3$ and $\alpha=1$, then $\left(I_{\lambda \alpha}\right)$ has a positive solution if and only if $\frac{1}{4} \lambda_{1}<\lambda<\lambda_{1}$, where $\lambda_{1}$ is the principal eigenvalue of $-\Delta$ with the homogeneous Dirichlet boundary condition ([2]). As the application of the result, we can prove the existence of a positive solution of the following problem:

$$
\left\{\begin{array}{l}
-\Delta u=g(x) u^{\frac{N+2}{N-2}} \text { in } \Omega, \\
(1-\alpha) \frac{\partial u}{\partial n}+\alpha u=0 \text { on } \partial \Omega,
\end{array}\right.
$$

if the function $g$ satisfies the above same condition. We also note that, if $\Omega$ is an open ball, $g=1$ in $\bar{\Omega}$ and $\alpha=1$, we have had the nonexistence result of any positive solution ([5]). The above difference between the existence and the non-existence may be appeared from the special properties of the function $g$ in the indefinite weight problems. 


\section{The main results}

We first recall some facts about how the method of eigencurves can be used to define principal eigenvalues. We define $\mu(\lambda, \alpha)$ by

$\mu(\lambda, \alpha)=\inf \left\{\int_{\Omega}\left(|\nabla u|^{2}-\lambda g u^{2}\right) d x+\frac{\alpha}{1-\alpha} \int_{\partial \Omega} u^{2} d S_{x}: u \in W^{1,2}(\Omega), \int_{\Omega} u^{2}=1\right\}$.

It can be shown in [1] that $\mu(0, \alpha)>0$ on $\alpha \in\left[\alpha_{0}, 1\right]$ where $\alpha_{0} \leq 0$ for some small negative, and the function $\lambda \longrightarrow \mu(\lambda, \alpha)$ is a concave function such that $\mu(0, \lambda) \rightarrow-\infty$ as $\lambda \rightarrow \pm \infty$. So it follows that $\lambda \rightarrow \mu(\lambda, \alpha)$ has exactly two zeros $\lambda_{\alpha}^{-}$and $\lambda_{\alpha}^{+}$, and those are principal eigenvalues for $\left(L_{\alpha}\right)$. Furthermore, the eigencurves $\lambda \rightarrow \mu(\lambda, \alpha)$ can be used to produce an equivalent norm for $W^{1,2}(\Omega)$ if $\alpha \neq 1$. In this case $\alpha=1$, we use the following function $\mu$ :

$$
\mu(\lambda)=\inf \left\{\int_{\Omega}\left[|\nabla|^{2}-\lambda g u^{2}\right] d x: u \in W_{0}^{1,2}(\Omega), \int_{\Omega} u^{2}=1\right\} .
$$

Lemma 2.1 ([4]). Suppose $\alpha \in(0,1)$ or that $\int_{\Omega} g d x \neq 0$ and $\alpha \in\left(\alpha_{0}, 0\right]$ so that $\left(L_{\alpha}\right)$ has principal eigenvalues $\lambda_{\alpha}^{-}$and $\lambda_{\alpha}^{+}$. For any $\lambda \in\left(\lambda_{\alpha}^{-}, \lambda_{\alpha}^{+}\right)$,

$$
\|u\|_{\lambda \alpha}=\left\{\int_{\Omega}\left[|\nabla u|^{2}-\lambda g u^{2}\right] d x+\frac{\alpha}{1-\alpha} \int_{\partial \Omega} u^{2} d S_{x}\right\}^{\frac{1}{2}}
$$

defines a norm in $W^{1,2}(\Omega)$ which is equivalent to the usual norm for $W^{1,2}(\Omega)$.

For the simplicity, we use the following function $K: \mathbb{R} \rightarrow \mathbb{R}$

$$
K(\alpha)=\left\{\begin{array}{cl}
0 & \text { if } \alpha=1 \\
\frac{\alpha}{1-\alpha} & \text { otherwise }
\end{array}\right.
$$

Lemma 2.2. Let $\lambda \in\left(\lambda_{\alpha}^{-}, \lambda_{\alpha}^{+}\right), \lambda \neq 0$ and let

$$
M_{\lambda \alpha}=\left\{u \in W^{1,2}(\Omega): u \neq 0,\left\langle J_{\lambda \alpha}^{\prime}(u), u\right\rangle=0\right\},
$$

Then $M_{\lambda \alpha}$ is a nonempty subset of $W^{1,2}(\Omega)$.

Proof. Since $g$ changes sign, we can choose a nonzero function $u_{0} \in W^{1,2}(\Omega)$ so that

$$
\int_{\Omega} g\left|u_{0}\right|^{p+2}>0
$$

Let

$$
t^{p}=\frac{\int_{\Omega}\left|\nabla u_{0}\right|^{2}-\lambda \int_{\Omega} g u_{0}^{2}+K(\alpha) \int_{\partial \Omega} u_{0}^{2} d S_{x}}{\lambda \int_{\Omega} g\left|u_{0}\right|^{p+2}} .
$$

Then $u=t u_{0} \in M_{\lambda \alpha}$. 
Definition 2.3. We define the following functions:

$$
\begin{aligned}
& K_{\lambda \alpha}^{+}=\inf \left\{\int_{\Omega}\left[|\nabla u|^{2}-\lambda g u^{2}\right]+K(\alpha) \int_{\partial \Omega} u^{2} d S_{x}: u \in W^{1,2}(\Omega), \int_{\Omega} g|u|^{p+2}=1\right\}, \\
& K_{\lambda \alpha}^{-}=\inf \left\{\int_{\Omega}\left[|\nabla u|^{2}-\lambda u^{2}\right]+K(\alpha) \int_{\partial \Omega} u^{2} d S_{x}: u \in W^{1,2,}(\Omega), \int_{\Omega} g|u|^{p+2}=-1\right\}, \\
& K_{0 \alpha}^{+}=\inf \left\{\int_{\Omega}|\nabla u|^{2}+K(\alpha) \int_{\partial \Omega} u^{2} d S_{x}: u \in W^{1,2}(\Omega), \int_{\Omega} g|u|^{p+2}=1\right\}
\end{aligned}
$$

and

$$
K_{0 \alpha}^{-}=\inf \left\{\int_{\Omega}|\nabla u|^{2}+K(\alpha) \int_{\partial \Omega} u^{2} d S_{x}: u \in W^{1,2}(\Omega), \int_{\Omega} g|u|^{p+2}=-1\right\} .
$$

Lemma 2.4. $K_{0 \alpha}^{-}>0$ and $K_{0 \alpha}^{+}>0$ if $\alpha \in(0,1]$.

Proof. Let $\alpha \neq 1$. We show that $K_{0 \alpha}^{+}>0$. If not, there is a sequence $u_{n} \in$ $W^{1,2}(\Omega)$ so that

$$
\lim _{n \rightarrow \infty}\left[\int_{\Omega}\left|\nabla u_{n}\right|^{2}+K(\alpha) \int_{\partial \Omega} u_{n}^{2} d S_{x}\right]=0 \text { and } \int_{\Omega} g\left|u_{n}\right|^{p+2}=1 .
$$

By the Sobolev embedding : $W^{1,2}(\Omega) \hookrightarrow L^{\frac{2 N}{N-2}}(\Omega)$, it is impossible.

The proof of $K_{0 \alpha}^{-}>0$ is exactly the same as the above.

By the similar method, we can prove that in the case $\alpha=1$.

Remark 2.5. Let $\alpha \in[0,1]$. We note that $K_{\lambda \alpha}^{-}$and $K_{\lambda \alpha}^{+}$are concave continuous curves on the interval $\left[\lambda_{\alpha}^{-}, \lambda_{\alpha}^{+}\right]$. Hence, $K_{\lambda \alpha}^{+} \leq K_{0 \alpha}^{+}$for all $\lambda \in\left[0, \lambda_{\alpha}^{+}\right]$and $K_{\lambda \alpha}^{-} \leq K_{0 \alpha}^{-}$for all $\lambda \in\left[\lambda_{\alpha}^{-}, 0\right]$. Furthermore, by the Sobolev embedding, the equivalent norm, and the relations between the principal eigenvalues and the function $g: \lambda \int_{\Omega} g \phi^{p+1} d x>0$ for all $p \geq 1$, where $\lambda \neq 0$ is a principal eigenvalue with corresponding positive principal eigenfunction $\phi([4])$, the following properties hold: (i) $K_{\lambda_{\alpha}^{-}}^{-}=K_{\lambda_{\alpha}^{+}}^{+}=0$, (ii) $K_{\lambda \alpha}^{-}>0$ if $\lambda \in\left(0, \lambda_{\alpha}^{+}\right)$and $K_{\lambda \alpha}^{+}>0$ if $\lambda \in\left(0, \lambda_{\alpha}^{+}\right)$.

Definitions and Remarks. Let $\lambda \in\left(\lambda_{\alpha}^{-}, \lambda_{\alpha}^{+}\right)$. We define the following sets:

$$
H_{\lambda}=\left\{u \in W^{1,2}(\Omega): \lambda \int_{\Omega} g|u|^{p+2}=1\right\} \text {. }
$$

Let $u \in H_{\lambda}$. Then $\|u\|_{\lambda \alpha}^{\frac{2}{p}} u \in M_{\lambda \alpha}$. If $u \in M_{\lambda \alpha}$, then $\|u\|_{\lambda \alpha}^{-\frac{2}{p+2}} u \in H_{\lambda}$. We define the functional $E_{\lambda \alpha}: H_{\lambda} \longrightarrow \mathbb{R}^{1}$ by

$$
E_{\lambda \alpha}(u)=\int_{\Omega}|\nabla u|^{2}-\lambda \int_{\Omega} g u^{2}+K(\alpha) \int_{\partial \Omega} u^{2} d S_{x} .
$$

Then we obtain

$$
E_{\lambda \alpha}(u)=\left[\frac{2(p+2)}{p} J_{\lambda \alpha}\left(\|u\|_{\lambda \alpha}^{\frac{2}{p}} u\right)\right]^{\frac{p}{p+2}}
$$


and

If we let

$$
J_{\lambda \alpha}(u)=\frac{p}{2(p+2)} E_{\lambda \alpha}\left(\|u\|_{\lambda \alpha}^{-\frac{2}{p+2}} u\right)^{\frac{p+2}{p}}
$$

$$
Q_{\lambda \alpha}=\inf E_{\lambda \alpha}\left(H_{\lambda}\right) \text { and } C_{\lambda \alpha}=\inf J_{\lambda \alpha}\left(M_{\lambda \alpha}\right),
$$

then by the simple calculation it follows that

$$
Q_{\lambda \alpha}=\left[\frac{2(p+2)}{p} C_{\lambda \alpha}\right]^{\frac{p}{p+2}} .
$$

This implies that if $\left\{u_{n}\right\}$ is a minimizing sequence of $E_{\lambda \alpha}$ on $H_{\lambda}$, then $\left\{\left\|u_{n}\right\|_{\lambda \alpha}^{\frac{2}{p}}\right.$ $\left.u_{n}\right\}$ is also a minimizing sequence of $J_{\lambda \alpha}$ on $M_{\lambda \alpha}$ and vice versa.

Remark 2.6. Let $\alpha \neq 1$. We can prove that $u=0$ is not a limit point of $M_{\lambda \alpha}$ if $\lambda_{\alpha}^{-}<\lambda<\lambda_{\alpha}^{+}$. To show that, we assume there is a sequence $\left\{u_{n}\right\}$ in $M_{\lambda \alpha}$ so that $\left\|u_{n}\right\|_{\lambda \alpha} \rightarrow 0$ as $n \rightarrow \infty$. From the Sobolev embedding: $W^{1,2}(\Omega) \hookrightarrow L^{\frac{2 N}{N-2}}(\Omega)$, the sequence $\left\{w_{n}\right\}$ which is defined by $w_{n}=\frac{u_{n}}{\left\|u_{n}\right\|_{\lambda \alpha}}$ is a bounded sequence in $L^{\frac{2 N}{N-2}}(\Omega)$. We hence have the following result:

$$
\begin{aligned}
0 & =\frac{\left\langle J_{\lambda \alpha}^{\prime}\left(u_{n}\right), u_{n}\right\rangle}{\left\|u_{n}\right\|_{\lambda}^{2}} \\
& =\frac{\int_{\Omega}\left|\nabla u_{n}\right|^{2}-\lambda \int_{\Omega} g u_{n}^{2}+K(\alpha) \int_{\partial \Omega} u_{n}^{2} d S_{x}}{\left\|u_{n}\right\|_{\lambda \alpha}^{2}}+\left(\left\|u_{n}\right\|_{\lambda \alpha}\right)^{p+2} \int_{\Omega} g\left|w_{n}\right|^{p+2} \rightarrow 1
\end{aligned}
$$

as $n \rightarrow \infty$, which leads to a contradiction.

We can also have the same argument for the case $\alpha=1$ by the similar method.

Lemma 2.7. Let $\alpha \in(0,1]$ or $\int_{\Omega} g d x \neq 0$ if $\alpha=0$. There are two positive numbers $\delta_{1}$ and $\delta_{2}$ such that for any $\lambda \in\left(\lambda_{\alpha}^{-}, \lambda_{\alpha}^{-}+\delta_{1}\right) \cup\left(\lambda_{\alpha}^{+}-\delta_{2}, \lambda_{\alpha}^{+}\right)$, if $\left\{u_{n}\right\}$ be a minimizing sequence of $J_{\lambda \alpha}$ on $M_{\lambda \alpha}$. Then

$$
\liminf _{n \rightarrow \infty}\left|\int_{\Omega} g u_{n}^{2}\right|>0
$$

Proof. Let $\varphi^{-}$and $\varphi^{+}$be the corresponding eigenfunctions to the principal eigenvalues $\lambda_{\alpha}^{-}$and $\lambda_{\alpha}^{+}$, respectively. We can assume that

$$
\int_{\Omega} g\left|\varphi^{-}\right|^{p+2}=-1, \quad \int_{\Omega} g\left|\varphi^{+}\right|^{p+2}=1 .
$$

(Lemma 3.1 in $[4]$ ). We also note that

Let

$$
\int_{\Omega} g\left(\varphi^{-}\right)^{2}<0, \quad \int_{\Omega} g\left(\varphi^{+}\right)^{2}>0
$$

$$
\delta_{2}=\lambda_{\alpha}^{+}-\frac{\int_{\Omega}\left|\nabla \varphi^{+}\right|^{2}-K_{0 \alpha}^{+}+K(\alpha) \int_{\partial \Omega}\left|\varphi^{+}\right|^{2} d S_{x}}{\int_{\Omega} g\left|\varphi^{+}\right|^{2}}=\frac{K_{0 \alpha}^{+}}{\int_{\Omega} g\left|\varphi^{+}\right|^{2}}
$$


Then for $\lambda \in\left(\lambda_{\alpha}^{+}-\delta_{2}, \lambda_{\alpha}^{+}\right)$and if $\left\{u_{n}\right\}$ is a minimizing sequence of $J_{\lambda \alpha}$ on $M_{\lambda \alpha}$, it is bounded in $W^{1,2}(\Omega)$, and then $u_{n} \rightarrow u$ weakly in $W^{1,2}(\Omega)$ and $u_{n} \rightarrow u$ strongly in $L^{2}(\Omega)$. We assume that $\lambda>0$. By the previous equality about minimums we know that $\left\{\left\|u_{n}\right\|_{\lambda \alpha}^{-\frac{2}{p+2}} u_{n}\right\}$ is a minimizing sequence of $E_{\lambda \alpha}$ on $H_{\lambda}$, and so there is a positive number $q$ such that

$$
\lim _{n \rightarrow \infty} \lambda^{\frac{2}{p+2}}\left\|u_{n}\right\|_{\lambda \alpha}^{-\frac{4}{p+2}}\left[\int_{\Omega}\left|\nabla u_{n}\right|^{2}-\lambda \int_{\Omega} g\left(u_{n}\right)^{2}+K(\alpha) \int_{\partial \Omega} u_{n}^{2} d S_{x}\right]<q<K_{0 \alpha}^{+}
$$

for some $g>0$. Since $\left\|u_{n}\right\|_{\lambda \alpha} \nrightarrow 0$ as $n \rightarrow \infty$, if $\int_{\Omega} g\left(u_{n}\right)^{2} \rightarrow 0$ as $n \rightarrow \infty$, we get

$$
K_{0 \alpha}^{+} \leq q<K_{0 \alpha}^{+},
$$

which leads to a contradiction. Therefore,

$$
\lim _{n \rightarrow \infty} \int_{\Omega} g u_{n}^{2} \neq 0
$$

Let

$$
\delta_{1}=\frac{\int_{\Omega}\left|\nabla \varphi^{-}\right|^{2}-K_{0 \alpha}^{-}+K(\alpha) \int_{\partial \Omega}\left|\varphi^{-}\right|^{2} d S_{x}}{\int_{\Omega} g\left|\varphi^{-}\right|^{2}}-\lambda_{\alpha}^{-}=-\frac{K_{0 \alpha}^{-}}{\int_{\Omega} g\left|\phi^{-}\right|^{2}} .
$$

For the value $\lambda \in\left(\lambda_{\alpha}^{-}, \lambda_{\alpha}^{-}+\delta_{1}\right)$ and $\lambda<0$, we can get the same results by the above methods.

This completes the proof.

We denote by $B_{\varepsilon}(X)$ the ball in a Hilbert space $X$ centered at 0 and of radius $\varepsilon$. We state the following:

Proposition 2.8 ([3], pp. 6). Let $J$ be a $C^{1}$-functional on a Hilbert space $X$ and let $M$ be a closed subset of $X$ verifying the following property:

For any $u \in M$ with $J^{\prime}(u) \neq 0$, there exists, for a small enough $\varepsilon>0$, a Fréchet differentiable function $s_{u}: B_{\varepsilon}(X) \longrightarrow \mathbb{R}^{1}$ such that, by setting $t_{u}(\delta)=$ $s_{u}\left(\delta \frac{J^{\prime}(\boldsymbol{u})}{\left\|J^{\prime}(\boldsymbol{u})\right\|}\right)$ for $0 \leq \delta \leq \varepsilon$, we have

$$
t_{u}(0)=1 \text { and } t_{u}(\delta)\left(u-\delta \frac{J^{\prime}(u)}{\left\|J^{\prime}(u)\right\|}\right) \in M .
$$

If $J$ is bounded below on $M$, then for any minimizing sequence $\left\{v_{n}\right\}$ in $M$ for $J$, there exists another minimizing sequence $\left\{u_{n}\right\}$ in $M$ of $J$ such that

$$
J\left(u_{n}\right) \leq J\left(v_{n}\right), \lim _{n \rightarrow \infty}\left\|u_{n}-v_{n}\right\|=0
$$

and

$$
\left\|J^{\prime}\left(u_{n}\right)\right\| \leq \frac{1}{n}\left(1+\| u_{n}||\left|t_{u_{n}}^{\prime}(0)\right|\right)+\left|t_{u_{n}}^{\prime}(0)\right|\left|\left\langle J^{\prime}\left(u_{n}\right), u_{n}\right\rangle\right|
$$

where $\langle$,$\rangle is the inner product in X$.

Proof. Let $C=\inf J(M)$. Use Ekeland's variational principle ([3]) to get a minimizing sequence $\left\{u_{n}\right\}$ in $M$ with the following properties: 
(i): $J\left(u_{n}\right) \leq J\left(v_{n}\right)<C+\frac{1}{n}$,

(ii): $\lim _{n \rightarrow \infty}\left\|u_{n}-v_{n}\right\|=0$,

(iii): $J(w) \geq J\left(u_{n}\right)-\frac{1}{n}\left\|w-u_{n}\right\|$ for all $w \in M$.

Let us assume $\left\|J^{\prime}\left(u_{n}\right)\right\|>0$ for $n$ large, since otherwise we are done. Apply the hypothesis on the set $M$ with $u=u_{n}$ to find $t_{n}(\delta)=s_{u_{n}}\left(\delta \frac{J^{\prime}\left(u_{n}\right)}{\left\|J^{\prime}\left(u_{n}\right)\right\|}\right)$ such that $w_{\delta}=t_{n}(\delta)\left(u_{n}-\delta \frac{J^{\prime}\left(u_{n}\right)}{\left\|J^{\prime}\left(u_{n}\right)\right\|}\right) \in M$ for all small enough $\delta \geq 0$.

Use now the mean value theorem to get

$$
\begin{aligned}
& \frac{1}{n}\left\|w_{\delta}-u_{n}\right\| \geq J\left(u_{n}\right)-J\left(w_{\delta}\right) \\
= & \left(1-t_{n}(\delta)\right)\left\langle J^{\prime}\left(w_{\delta}\right), u_{n}\right\rangle+\delta t_{n}(\delta)\left\langle J^{\prime}\left(w_{\delta}\right), \frac{J^{\prime}\left(u_{n}\right)}{\left\|J^{\prime}\left(u_{n}\right)\right\|}\right\rangle+o(\delta),
\end{aligned}
$$

where $\frac{\mathrm{o}(\delta)}{\delta} \rightarrow 0$ as $\delta \rightarrow 0$. Dividing by $\delta>0$ and passing to the limit as $\delta \rightarrow 0$ we derive

$$
\frac{1}{n}\left(1+\left|t_{n}^{\prime}(0)\right| \| u_{n}||\right) \geq-t_{n}^{\prime}(0)\left\langle J^{\prime}\left(u_{n}\right), u_{n}\right\rangle+\left\|J^{\prime}\left(u_{n}\right)\right\|,
$$

which is our claim.

Lemma 2.9. Let $\alpha \in(0,1]$ or that $\int_{\Omega} g d x \neq 0$ for $\alpha=0$. Given $\lambda \epsilon$ $\left(\lambda_{\alpha}^{-}, \lambda_{\alpha}^{+}\right), \lambda \neq 0, J_{\lambda \alpha}$ is bounded below on $M_{\lambda \alpha}$ and there exists a minimizing sequence $\left\{u_{n}\right\}$ of $J_{\lambda \alpha}$ on $M_{\lambda \alpha}$ so that

$$
\lim _{n \rightarrow \infty}\left\|J_{\lambda \alpha}^{\prime}\left(u_{n}\right)\right\|_{\lambda \alpha}=0
$$

and

$$
\lim _{n \rightarrow \infty} J_{\lambda \alpha}\left(u_{n}\right)=\inf J_{\lambda \alpha}\left(M_{\lambda \alpha}\right) .
$$

Proof. Let $\lambda \in\left(\lambda_{\alpha}^{-}, \lambda_{\alpha}^{+}\right)$and let $\lambda \neq 0$. We show that $J_{\lambda \alpha}$ is bounded below on $M_{\lambda \alpha}$. In fact, the following can be checked easily: if $u \in M_{\lambda \alpha}$, then

$$
\lambda \int_{\Omega} g|u|^{p+2}>0
$$

and

$$
J_{\lambda \alpha}(u)=\frac{p \lambda}{2(p+2)} \int_{\Omega} g|u|^{p+2} .
$$

Let $u \in M_{\lambda \alpha}$. Define $G: \mathbb{R}^{1} \times W^{1,2}(\Omega) \longrightarrow \mathbb{R}^{1}$ by $G(s, w)=\Phi_{\lambda \alpha}(s(u-w))$, where $\Phi_{\lambda \alpha}: W^{1,2}(\Omega) \longrightarrow \mathbb{R}^{1}$ is a functional defined by

$$
\Phi_{\lambda \alpha}(u)=\int_{\Omega}|\nabla u|^{2}-\lambda \int_{\Omega} g u^{2}-\lambda \int_{\Omega} g|u|^{p+2}+K(\alpha) \int_{\partial \Omega} u^{2} d S_{x} .
$$


Then $G(1,0)=0$ and

$$
\begin{aligned}
& \frac{d}{d s} G(1,0) \\
= & 2 \int_{\Omega}|\nabla u|^{2}-2 \lambda \int_{\Omega} g u^{2}-\lambda(p+2) \int_{\Omega} g|u|^{p+2}+2 K(\alpha) \int_{\partial \Omega} u^{2} d S_{x} \\
= & -p\left(\int_{\Omega}\left[|\nabla u|^{2}-\lambda g u^{2}\right]+K(\alpha) \int_{\partial \Omega} u^{2} d S_{x}\right) \neq 0 .
\end{aligned}
$$

Hence, we can apply the Implicit Function Theorem at $(1,0)$ and get that for $\delta>0$ small enough, there exists a differentiable function

$$
s_{u}: B_{\delta}\left(W^{1,2}(\Omega)\right) \rightarrow \mathbb{R}^{1}
$$

such that $s_{u}(0)=1, s_{u}(w)(u-w) \in M_{\lambda \alpha}$, and

$$
\left\langle s_{u}^{\prime}(0), w\right\rangle=\frac{\left\langle\Phi_{\lambda \alpha}^{\prime}(u), w\right\rangle}{\left\langle\Phi_{\lambda \alpha}^{\prime}(u), u\right\rangle}
$$

for all $w \in B_{\delta}\left(W^{1,2}(\Omega)\right)$. From the identification of duality to the Hilbert space $W^{1,2}(\Omega)$, we let

$$
w_{u}=\frac{J_{\lambda \alpha}^{\prime}(u)}{\left\|J_{\lambda \alpha}^{\prime}(u)\right\|_{\lambda \alpha}} \text { and } t_{u}(\rho)=s_{u}\left(\rho w_{u}\right)
$$

for all $0 \leq \rho \leq \delta$. Then $t_{u}(0)=1$ and

$$
t_{u}(\rho)\left(u-\rho w_{u}\right)=s_{u}\left(\rho w_{u}\right)\left(u-\rho w_{u}\right) \in M_{\lambda \alpha} .
$$

From Proposition 2.8, there is a minimizing sequence $\left\{u_{n}\right\}$ of $J_{\lambda \alpha}$ on $M_{\lambda \alpha}$ so that

$$
J_{\lambda \alpha}\left(u_{n}\right) \leq J_{\lambda \alpha}\left(v_{n}\right)<\inf J_{\lambda \alpha}\left(M_{\lambda \alpha}\right)+\frac{1}{n}, \lim _{n \rightarrow \infty}\left\|u_{n}-v_{n}\right\|_{\lambda \alpha}=0
$$

and

$$
\left\|J_{\lambda \alpha}^{\prime}\left(u_{n}\right)\right\|_{\lambda \alpha} \leq \frac{1}{n}\left(1+\left|t_{u_{n}}^{\prime}(0)\right||| u_{n} \|_{\lambda \alpha}\right)+\left|t_{u_{n}}^{\prime}(0)\right|\left|\left\langle J_{\lambda \alpha}^{\prime}\left(u_{n}\right), u_{n}\right\rangle\right|
$$

Since $J_{\lambda \alpha}\left(u_{n}\right)=\frac{\lambda p}{2(p+2)}\left\|u_{n}\right\|_{\lambda \alpha}^{2}$, so the sequence $\left\{u_{n}\right\}$ is bounded in $W^{1,2}(\Omega)$. Let $\left\|u_{n}\right\|_{\lambda \alpha} \leq C_{1}$ for all $n$. Then

$$
\left\|J_{\lambda \alpha}^{\prime}\left(u_{n}\right)\right\|_{\lambda \alpha} \leq \frac{1}{n}\left(1+\left|t_{u_{n}}^{\prime}(0)\right| C_{1}\right)
$$

Since

$$
\left|t_{u_{n}}^{\prime}(0)\right|=\frac{\left|\left\langle\Phi_{\lambda \alpha}^{\prime}\left(u_{n}\right), w_{n}\right\rangle\right|}{p\left\|u_{n}\right\|_{\lambda \alpha}^{2}}
$$


where $w_{n}=w_{u_{n}}$, and $\lim _{n \rightarrow \infty} \inf || u_{n} \|_{\lambda \alpha}>0$, if we show that $\left|t_{u_{n}}^{\prime}(0)\right|$ is uniformly bounded on $n$, we are done. In fact, we have the following inequality

$$
\begin{aligned}
& \left|\left\langle\Phi_{\lambda \alpha}^{\prime}\left(u_{n}\right), w_{n}\right\rangle\right| \\
\leq & 2 \int_{\Omega}\left|\nabla u_{n} \cdot \nabla w_{n}\right|+2 \lambda \int_{\Omega}\left|u_{n} w_{n}\right|+\lambda(p+2) \int_{\Omega}|g|\left|u_{n}\right|^{p+1}\left|w_{n}\right| \\
& +2 K(\alpha) \int_{\partial \Omega} u_{n} w_{n} d S_{x} .
\end{aligned}
$$

From the well-known Sobolev embedding theorem, $\left\|w_{n}\right\|_{\lambda \alpha}=1$ for all $n$, and Hölder inequality, we have two positive constants $C_{2}$ and $C_{3}$ so that

$$
\left|\left\langle\Phi_{\lambda \alpha}^{\prime}\left(u_{n}\right), w_{n}\right\rangle\right| \leq C_{2}\left\|u_{n}\right\|_{\lambda \alpha}+C_{3} .
$$

Since $\left\{u_{n}\right\}$ is a bounded sequence in $W^{1,2}(\Omega)$, so is $\left\langle\Phi_{\lambda \alpha}^{\prime}\left(u_{n}\right), w_{n}\right\rangle$ on $n$. Therefore, we can conclude that

$$
\lim _{n \rightarrow \infty}\left\|J_{\lambda \alpha}^{\prime}\left(u_{n}\right)\right\|_{\lambda \alpha}=0
$$

Clearly, we note that

$$
\lim _{n \rightarrow \infty} J_{\lambda \alpha}\left(u_{n}\right)=\inf J_{\lambda \alpha}\left(M_{\lambda \alpha}\right)
$$

Theorem 2.10. Let $\alpha \in(0,1]$ or that $\int_{\Omega} g d x \neq 0$ for $\alpha=0$. For any $\lambda \in$ $\left(\lambda_{\alpha}^{-}, \lambda_{\alpha}^{-}+\delta_{1}\right) \cup\left(\lambda_{\alpha}^{+}-\delta_{2}, \lambda_{\alpha}^{+}\right), \lambda \neq 0$, the problem $\left(I_{\lambda \alpha}\right)$ has a positive solution.

Proof. Let

$$
c=\inf J_{\lambda \alpha}\left(M_{\lambda \alpha}\right)
$$

and let $\left\{u_{n}\right\}$ be a sequence in $M_{\lambda \alpha}$ such that

$$
\lim _{n \rightarrow \infty} J_{\lambda \alpha}\left(u_{n}\right)=c .
$$

By Lemma 2.9, we can assume that

$$
\lim _{n \rightarrow \infty}\left\|J_{\lambda \alpha}^{\prime}\left(u_{n}\right)\right\|_{\lambda \alpha}=0 .
$$

Then $\left\{u_{n}\right\}$ is bounded and we can find a weak limit point $u$ of the sequence in $W^{1,2}(\Omega)$. We can also assume that $\left\{u_{n}\right\}$ converges weakly to $u$ and, by the Rellich-Kondrakov Theorem $([3])$, that $u_{n} \rightarrow u$ strongly in $L^{q}(\Omega)$ for all $q<\frac{2 N}{N-2}$. In particular, for any $v \in W^{1,2}(\Omega)$,

$$
\left\langle J_{\lambda \alpha}^{\prime}\left(u_{n}\right), v\right\rangle=\int_{\Omega} \nabla u_{n} \cdot \nabla v-\lambda \int_{\Omega} g u_{n} v-\lambda \int_{\Omega} g u_{n}\left|u_{n}\right|^{p} v+K(\alpha) \int_{\partial \Omega} u_{n} v d S_{x},
$$

which converges as $n \rightarrow \infty$ to

$$
\int_{\Omega}\left(\nabla u \cdot \nabla v-\lambda g u v-\lambda g u|u|^{p} v\right) d x+K(\alpha) \int_{\partial \Omega} u v d S_{x}=\left\langle J_{\lambda \alpha}^{\prime}(u), v\right\rangle .
$$


Hence, $\left\langle J_{\lambda \alpha}^{\prime}(u), v\right\rangle=0$ for all $v \in W^{1,2}(\Omega)$ which means that $u$ is a weak solution for $\left(I_{\lambda \alpha}\right)$. In particular, $\left\langle J_{\lambda \alpha}^{\prime}(u), u\right\rangle=0$. Since $\liminf _{n \rightarrow \infty}\left|\int_{\Omega} g u_{n}^{2}\right|>0$ by Lemma 2.7, we have that $u \neq 0$. Therefore, $u \in M_{\lambda \alpha}$.

Since $J_{\lambda \alpha}$ is weakly lower semi-continuous, we get

$$
c \leq J_{\lambda \alpha}(u) \leq \lim _{n \rightarrow \infty} J_{\lambda \alpha}\left(u_{n}\right)=c .
$$

It follows that $J_{\lambda \alpha}(u)=c$ and that $\left\|u_{n}\right\|_{\lambda \alpha} \rightarrow\|u\|_{\lambda \alpha}$ which implies that $u_{n} \rightarrow u$ strongly in $W^{1,2}(\Omega)$. Since $J_{\lambda \alpha}^{\prime}$ is continuous at $u$, we get $J_{\lambda \alpha}^{\prime}(u)=0$.

The positivity of $u$ is clear from the equality $J_{\lambda \alpha}(u)=J_{\lambda \alpha}(|u|)$.

This completes the proof.

Theorem 2.11. Let $\alpha \in(0,1]$ or that $\int_{\Omega} g d x \neq 0$ for $\alpha=0$. If $g(x)<0$ for all $x \in \partial \Omega$, for any $\lambda \in\left(0, \lambda_{\alpha}^{+}\right)$, the problem $\left(I_{\lambda \alpha}\right)$ has a positive solution.

Proof. By Theorem 2.10, we have a positive solution $u_{\lambda}$ of the problem:

$$
\left\{\begin{array}{l}
-\Delta u=\lambda g(x) u+g(x) u|u|^{p} \text { in } \Omega \\
(1-\alpha) \frac{\partial u}{\partial n}+\alpha u=0 \text { on } \partial \Omega
\end{array}\right.
$$

for $\lambda \in\left(\lambda_{\alpha}^{-}+\delta_{2}, \lambda_{\alpha}^{-}\right) \cup\left(\lambda_{\alpha}^{+}-\delta_{1}, \lambda_{\alpha}^{+}\right)$.

Let $0<\lambda<\lambda_{\alpha}^{+}$and let $\left\{u_{n}\right\}$ be a minimizing sequence of $J_{\lambda \alpha}$ in $M_{\lambda \alpha}$. If $K_{\lambda \alpha}^{+}<K_{0 \alpha}^{+}$on $\left(0, \lambda_{\alpha}^{+}\right)$, we can have the same inequality about the limit $\liminf _{n \rightarrow \infty}\left|\int_{\Omega} g u_{n}^{2}\right|>0$ by the same method in Lemma 2.7, and so using the same method in Theorem 2.10 we have the existence of a positive solution of $\left(I_{\lambda \alpha}\right)$. We note that $K_{\lambda \alpha}^{+} \leq K_{0 \alpha}^{+}$for all $\lambda \in\left(0, \lambda_{\alpha}^{+}\right)$.

Suppose that there is $\lambda_{0}$ so that $0<\lambda_{0}<\lambda_{\alpha}^{+}$and $K_{\lambda_{0} \alpha}^{+}=K_{0 \alpha}^{+}$and $K_{\lambda \alpha}^{+}<$ $K_{0 \alpha}^{+}$on $\left(\lambda_{0}, \lambda_{\alpha}^{+}\right)$. Let $u_{\lambda}$ be the positive minimizer of the functional $J_{\lambda \alpha}$ on $M_{\lambda \alpha}$ for $\lambda \in\left(\lambda_{0}, \lambda_{\alpha}^{+}\right)$. Let

$$
t_{\lambda}^{p}=\frac{\lambda \int_{\Omega} g u_{\lambda}^{p+2}+\left(\lambda-\lambda_{0}\right) \int_{\Omega} g u_{\lambda}^{2}+K(\alpha) \int_{\partial \Omega} u^{2} d S_{x}}{\lambda_{0} \int_{\Omega} g u_{\lambda}^{p+2}} .
$$

Then $t_{\lambda} u_{\lambda} \in M_{\lambda_{0} \alpha}$, and

$$
J_{\lambda_{0} \alpha}\left(t_{\lambda} u_{\lambda}\right)=t_{\lambda}^{2}\left[J_{\lambda \alpha}\left(u_{\lambda}\right)+\frac{p}{2(p+2)}\left(\lambda-\lambda_{0}\right) \int_{\Omega} g u_{\lambda}^{2}\right]
$$

As the previous calculation in Remark, we note that

$$
\inf _{\lambda \rightarrow \lambda_{0}} \int_{\Omega} g u_{\lambda}^{p+2}>0
$$

and we also note that

$$
\liminf _{\lambda \rightarrow \lambda_{0}} J_{\lambda \alpha}\left(u_{\lambda}\right)<\infty
$$


implies that

$$
\liminf _{\lambda \rightarrow \lambda_{0}}\left|\int_{\Omega} g u_{\lambda}^{2}\right| \neq \infty
$$

and hence, $t_{\lambda} \rightarrow 1$ as $\lambda \rightarrow \lambda_{0}$. Since $\left\{t_{\lambda} u_{\lambda}\right\}$ is a minimizing sequence of $J_{\lambda_{0} \alpha}$ as $\lambda \rightarrow \lambda_{0}$, we get the weak limit $u_{\lambda_{0}}$ of $u_{\lambda}$ so that

$$
\lim _{\lambda \rightarrow \lambda_{0}} t_{\lambda} u_{\lambda}=u_{\lambda_{0}} \text { in } L^{2}(\Omega) .
$$

If $u_{\lambda_{0}} \neq 0$, we know that it is the minimizer of $J_{\lambda_{0} \alpha}$ and is the positive solution of the above boundary value problem with respect to $\lambda_{0}$. Let

$$
v_{\lambda}=\lambda^{\frac{1}{p+2}}\left\|u_{\lambda}\right\|_{\lambda \alpha}^{-\frac{2}{p+2}} u_{\lambda}
$$

Then

and

$$
\begin{gathered}
K_{\lambda \alpha}^{+}=\int_{\Omega}\left|\nabla v_{\lambda}\right|^{2}-\lambda \int_{\Omega} g\left(v_{\lambda}\right)^{2}+K(\alpha) \int_{\partial \Omega} v_{\lambda}^{2} d S_{x} \\
K_{\lambda_{0} \alpha}^{+}=\int_{\Omega}\left|\nabla v_{\lambda_{0}}\right|^{2}-\lambda_{0} \int_{\Omega} g\left(v_{\lambda_{0}}\right)^{2}+K(\alpha) \int_{\partial \Omega} v_{\lambda_{0}}^{2} d S_{x}
\end{gathered}
$$

$$
-\int_{\Omega} g\left(v_{\lambda}\right)^{2} \leq \frac{K_{\lambda \alpha}^{+}-K_{\lambda_{0} \alpha}^{+}}{\lambda-\lambda_{0}} \leq-\int_{\Omega} g\left(v_{\lambda_{0}}\right)^{2} .
$$

Taking the limit on the both sides as $\lambda \rightarrow \lambda_{0}$, we get

$$
\frac{d K_{\lambda \alpha}^{+}}{d \lambda}\left(\lambda_{0}\right)=-\int_{\Omega} g\left(v_{\lambda_{0}}\right)^{2}
$$

Hence, $K_{\lambda \alpha}^{+}$is differentiable at $\lambda=\lambda_{0}$, and so $\int_{\Omega} g\left(v_{\lambda_{0}}\right)^{2}=0$. Since

$$
K_{\lambda_{0} \alpha}^{+}=K_{0 \alpha}^{+}=\int_{\Omega}\left|\nabla v_{\lambda_{0}}\right|^{2}+K(\alpha) \int_{\partial \Omega} v_{\lambda_{0}}^{2} d S_{x},
$$

so $v_{\lambda_{0}}$ is also a positive solution of the problem:

$$
\left\{\begin{array}{l}
-\Delta u=g(x) u|u|^{p} \text { in } \Omega, \\
(1-\alpha) \frac{\partial u}{\partial n}+\alpha u=0 \text { on } \partial \Omega,
\end{array}\right.
$$

which leads to a contradiction.

Let $u_{\lambda_{0}}=0$. The $u_{\lambda} \rightarrow 0$ a.e. in $\Omega$ as $\lambda \rightarrow \lambda_{0}$. By Harnack Inequality we can argue that $u_{\lambda} \rightarrow 0$ uniformly on any compact subset of $\Omega$. Also by the Maximum Principle $\left\{u_{\lambda}\right\}$ is uniformly bounded on any neighborhood of $\partial \Omega$. Therefore, $u_{\lambda} \rightarrow 0$ uniformly bounded in $\bar{\Omega}$, and then by the Lebesgue dominated convergence Theorem

$$
1=\lim _{\lambda \rightarrow \lambda_{0}}\left\|u_{\lambda}\right\|_{\lambda \alpha}^{-2} \int_{\Omega} g\left|u_{\lambda}\right|^{p+2}=0
$$

since $\left\|u_{\lambda}\right\|_{\lambda \alpha} \nrightarrow 0$ as $\lambda \rightarrow \lambda_{0}$, which also leads to a contradiction.

This completes the proof. 
Corollary 2.12. Let $\alpha \in(0,1]$ or that $\int_{\Omega} g d x \neq 0$ for $\alpha=0$. If $g$ satisfies the condition in Theorem 2.11, then the following problem:

$$
\left\{\begin{array}{l}
-\Delta u=g(x) u|u|^{\frac{4}{N-2}} \text { in } \Omega, \\
(1-\alpha) \frac{\partial u}{\partial n}+\alpha u=0 \text { on } \partial \Omega
\end{array}\right.
$$

has a positive solution.

Proof. With the result of Theorem 2.11 if we let $\lambda_{0}=0$, the proof for the convergence of a minimizing sequence of the functional:

$$
J(u)=\frac{1}{2} \int_{\Omega}|\nabla u|^{2}-\frac{1}{p+2} \int_{\Omega} g|u|^{p+2} d x+2 K(\alpha) \int_{\partial \Omega} u^{2} d S_{x}
$$

on the Nehari manifold

$$
\left\{u \in W^{1,2}(\Omega): u \neq 0, \int_{\Omega}|\nabla u|^{2}-\int_{\Omega} g|u|^{p+2}+K(\alpha) \int_{\partial \Omega} u^{2} d S_{x}=0\right\}
$$

can be produced by the one of Theorem 2.11 .

Acknowledgement. This work was supported by grant No. R05-2002-00000605-0 from the Basic Research Program of the Korea Science and Engineering Foundation.

\title{
References
}

[1] G. Afrouzi and K. Brown, On principal eigenvalues for boundary value problems with indefinite weight and Robin boundary conditions, Proc. Amer. Math. Soc. 127 (1999), no. $1,125-130$.

[2] H. Brezis and L. Nirenberg, Positive solutions of nonlinear elliptic equations involving critical Sobolev exponents, Comm. Pure Appl. Math. 36 (1983), no. 4, 437-477.

[3] N. Ghoussoub, Duality and perturbation methods in critical point theory, With appendices by David Robinson. Cambridge Tracts in Mathematics, 107. Cambridge University Press, Cambridge, 1993.

[4] B. Ko and K. Brown, The existence of positive solutions for a class of indefinite weight semilinear elliptic boundary value problems, Nonlinear Anal. 39 (2000), no. 5, Ser. A: Theory Methods, 587-597.

[5] R. Pohoz̃aev, Eigenfunctions on the equation $\Delta u+\lambda f(u)=0$, Soviet Math. Dokl. 6 (1965), 1408-1411.

\author{
BONGSOO KO \\ DEPARTMENT OF MATHEMATICS EDUCATION \\ Educational Research Institute \\ Cheju National University \\ Cheju 690-756, Korea \\ E-mail address: bsko@cheju.cheju.ac.kr \\ SEUNGPIL Kang \\ Department of Mathematics Education \\ EdUCational Research Institute \\ Cheju National University \\ CheJu 690-756, KoreA
}

